\title{
Analysis of harmonic resonance mechanism of PV power plant
}

\author{
Shi Jing ${ }^{*}$, Wang Zhimin, Huang Zhonghua and Qi Yanshou \\ Qinghai Electric Research Technology Co. Ltd., Xining, China
}

\begin{abstract}
Great amount of PV inverters connected with the PV power plant causes the power system to become electronically power and affects the operation mode of the traditional power system. Highfrequency switching characteristics of PV inverters carry a large number of harmonic components, even lead to the harmonic resonance of a multi-machine parallel system. In the paper, based on physical mechanism of power electronics, a high-order model of harmonic impedance of a typical PV power plant is established, and harmonic resonance mechanism in parallel operation of PV power plant is analyzed, as well as key factors.
\end{abstract}

\section{Introduction}

When a photovoltaic power plant connected to the power system, with power electronic equipment (PV inverters), it causes that power system to be electronically electronicized, affecting the operation mode of the traditional power system, and at the same time, new problems due to the particularity of the power electronic equipment[1]. Most of large-scale photovoltaic power plants in Qinghai Province, Northwest of China, contain tens to hundreds of inverters, and the power plant structure operates in parallel with multiple inverters. Since the high-frequency switching characteristics of inverters carry a large amount of harmonic components, in severe cases, PV power plant has been harmonic resonance, and there have been many such accidents.

At present, the harmonic resonance suppression technology for power electronic equipment mainly focuses on control strategies and modulation strategies. The literature [2] proposed an active damping scheme that replaces the actual damping resistance with a virtual resistance control algorithm, thereby replacing the actual passive damping resistance with a control algorithm. The literature [3] proposed to construct a link with negative resonance peak characteristics in control system and counteract positive resonance peak generated by the LCL filter. The literature [4] solved the switching angle of power device offline or online by specifying the amplitude of the specified sub-harmonic equal to zero, thereby eliminating the specific sub-harmonic of the output phase voltage of the inverter.

Normally, literatures aim to control the harmonic resonance of a single converter. It is rarely associated with the multi-inverter system, combined with the structure and link impedance of the entire multi-converter grid-connected system [5-7].

In this paper, a large-scale photovoltaic power plant is taken as the research object, and a high-order harmonic impedance model of multi-power electronic equipment grid-connected system is established to analyze the harmonic resonance mechanism of PV power plant.

\section{Harmonic impedance model of PV inverter}

The DC side shunt regulator capacitor of the PV inverter is inverted into three-phase bridge circuit, with a series of high frequency and discrete pulse voltages. The bridge voltage contains a large number of higher harmonics, and finally merged through the output filter and grid side.

\subsection{Mathematical model of LCL filter}

The typical circuit topology of LCL filter of PV inverter is shown in Fig.1.

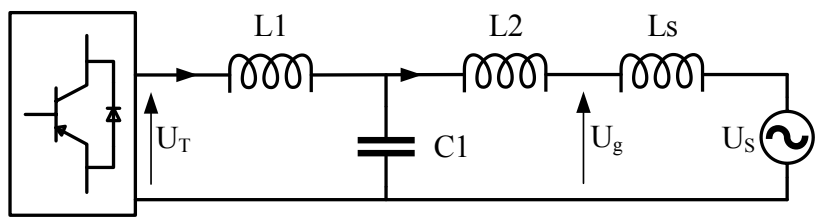

Fig. 1. PV inverter filter circuit topology.

According to Kirchhoff's law, the differential equations of $\mathrm{AC}$ side in the stationary coordinate system can be obtained:

$$
\left\{\begin{array}{l}
L_{1} \frac{d \dot{I}_{1}}{d t}=\dot{U}_{\text {inv }}-\dot{U}_{\mathrm{c}} \\
C_{1} \frac{d \dot{U}_{\mathrm{c}}}{d t}=\dot{I}_{1}-\dot{I}_{2} \\
L_{2} \frac{d \dot{I}_{\mathrm{g}}}{d t}=\dot{U}_{\mathrm{c}}-\dot{U}_{\mathrm{g}}
\end{array}\right.
$$

*Corresponding author: 67239861@qq.com 
According to the coordinate transformation theory, (1) can be transformed into:

$$
\left\{\begin{array}{l}
L_{1} \frac{d I_{1 \mathrm{~d}}}{d t}=U_{\mathrm{Td}}-U_{\mathrm{cd}}+\omega L_{1} I_{1 \mathrm{q}} \\
L_{1} \frac{d I_{1 \mathrm{q}}}{d t}=U_{\mathrm{Tq}}-U_{\mathrm{cq}}-\omega L_{1} I_{1 \mathrm{~d}} \\
C_{1} \frac{d U_{\mathrm{cd}}}{d t}=I_{1 \mathrm{~d}}-I_{\mathrm{gd}}+\omega C_{1} U_{\mathrm{cq}} \\
C_{1} \frac{d U_{\mathrm{cq}}}{d t}=I_{1 \mathrm{q}}-I_{\mathrm{gq}}-\omega C_{1} U_{\mathrm{cd}} \\
L_{2} \frac{d I_{\mathrm{gd}}}{d t}=U_{\mathrm{cd}}-U_{\mathrm{gd}}+\omega L_{2} I_{\mathrm{gq}} \\
L_{2} \frac{d I_{\mathrm{gq}}}{d t}=U_{\mathrm{cq}}-U_{\mathrm{gq}}-\omega L_{2} I_{\mathrm{gd}}
\end{array}\right.
$$

By performing the Laplace transform on (2), the impedance mathematical model of LCL filter can be obtained, which is a set of equations of multiple input multiple output nonlinear multivariable coupling.

\subsection{Mathematical model of inner-loop controller}

Normally, the PV inverter uses double-loop control, and its inner loop control is used to complete the output current control.

Under the $d q$ rotating coordinate system, the innerloop controller generally adopts a feedforward decoupling strategy, including a proportional integral controller and a feedforward decoupling link. The $d$-axis modulation degree $m_{\mathrm{d}}$ could be obtained by inputting the difference between the active control command $I_{\text {dref }}$ and the measured value $I_{\mathrm{d}}$ of the inner-loop to the $d$-axis PI controller, with adding the feedforward compensation and the decoupling compensation, as shown in Fig. 2.

\subsection{Norton equivalent model of PV inverter}

The following relationship can be obtained from Fig. 2:

$$
\left\{\begin{array}{l}
{\left[I_{\mathrm{gref}}(s)-I_{\mathrm{g}}(s)\right] G_{\mathrm{PI}}(s)=U_{\mathrm{T}}^{\prime}(s)} \\
U_{\mathrm{T}}(s)=K_{\mathrm{PWM}}\left[U_{\mathrm{g}}(s) G_{\mathrm{in}}(s)+U_{\mathrm{T}}^{\prime}(s)-K_{\mathrm{in}} I_{\mathrm{c}}(s)\right] \\
I_{\mathrm{c}}(s)=\left[U_{\mathrm{T}}(s)-U_{\mathrm{c}}(s)\right] \frac{1}{s L_{1}}-I_{\mathrm{g}}(s) \\
I_{\mathrm{c}}(s) \frac{1}{s C_{1}}=U_{\mathrm{c}}(s) \\
I_{\mathrm{g}}(s)=\left[U_{\mathrm{c}}(s)-U_{\mathrm{g}}(s)\right] \frac{1}{s L_{2}}
\end{array}\right.
$$

Furthermore, the output admittance of the converter is obtained:

$$
\begin{aligned}
Y_{\text {con }}(s) & =-\frac{I_{\mathrm{g}}(s)}{U_{\mathrm{g}}(s)} \\
& =\frac{K_{\mathrm{PWM}} K_{C} S C_{1}+1+s^{2} L_{1} C_{1}-K_{\mathrm{PWM}} G_{i n}(s)}{K_{\mathrm{PWM}} K_{C} s^{2} L_{2} C_{1}+s L_{2}+s^{3} L_{1} L_{2} C_{1}+K_{\mathrm{PWM}} G_{\mathrm{PI}}(s)+s L_{1}}
\end{aligned}
$$

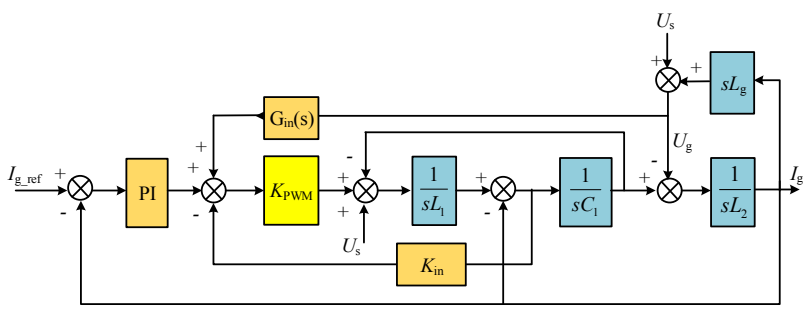

Fig. 2. Model of filter and inner loop controller.

\section{Harmonic impedance model of PV inverters and Analytical method}

Learn from the traditional power network analysis method, the impedance network model of multi-power electronic equipment is established firstly, and the harmonic characteristics of multi-power electronic equipment are analysed by the node network equations.

\subsection{System network equation}

Using the symmetrical component method, the $A, B$ and $C$ three-phase power networks can be transformed into positive, negative, and zero-sequence networks. Set the three-phase admittance network equations:

$$
I^{(a b c)}=Y^{(a b c)} U^{(a b c)}
$$

Where, $\boldsymbol{U}^{(\mathbf{a b c})}$ is the network node three-phase voltage column vector, $\boldsymbol{I}^{(\mathbf{a b c})}$ is the network node three-phase injection current column vector, and $\boldsymbol{Y}^{(\mathbf{a b c})}$ is the network node three-phase admittance matrix.

\subsection{Node admittance matrix}

In (5), $\boldsymbol{Y}^{(\mathbf{a b c})}$ is set:

$$
Y=\left[\begin{array}{cccc}
Y_{11} & Y_{12} & \cdots & Y_{1 n} \\
Y_{21} & Y_{22} & \cdots & Y_{2 n} \\
\vdots & \vdots & \ddots & \vdots \\
Y_{n 1} & Y_{n 2} & \cdots & Y_{n n}
\end{array}\right]
$$

Where, the order of the admittance matrix is equal to the number of nodes of the power network, and the diagonal elements $Y_{\text {ii }}$ of the admittance matrix, the self-admittance of each node, is equal to the sum of the admittances of the branches connected to the corresponding nodes:

$$
Y_{i i}=\sum y_{i j}
$$

Where, $y_{\mathrm{ij}}$ is reciprocal of the impedance of the path between node $i$ and node $j$.

The number of non-zero elements in the non-diagonal elements of each row of the admittance matrix is equal to the number of ungrounded branches connected to the corresponding nodes, and the non-diagonal elements $Y_{\mathrm{ij}}$ of the admittance matrix is equal to the negative 
admittance of the branch between the node $i$ and the node $j$.

$$
Y_{i j}=-\frac{1}{z_{i j}}=-y_{i j}
$$

Therefore, the admittance matrix can be established based on PV power plant network topology.

\subsection{Harmonic power flow method}

Harmonic power flow calculation is to solve the harmonic voltage of each node, according to the network equation composed of the harmonic current injected by each harmonic source node and the harmonic admittance matrix of the network, as following:

$$
\mathbf{U}(h)=\mathbf{Z}(h) \mathbf{I}(h)
$$

Where, $\boldsymbol{U}(h)$ is the node harmonic voltage vector, $\boldsymbol{Z}(h)$ is the node impedance matrix, $\boldsymbol{Z}(h)=\boldsymbol{Y}^{-1}(h)$, and $\boldsymbol{Y}(h)$ is the node admittance matrix; $\boldsymbol{I}(h)$ is the node injection harmonic current vector.

$\boldsymbol{I}(h)$ is the $h$-th harmonic injection current vector of each node. The non-zero element is the $h$-th harmonic injection current $I_{\mathrm{i}}(h)$ of each harmonic source, and the element without the harmonic source is zero, and the components to which they are connected are incorporated into the admittance matrix in the form of their equivalent admittance. As the harmonic current injection mode be known, the harmonic bus voltage can be obtained, and the corresponding harmonic line current can also be obtained.

It is noticed that harmonic network parameters used in the calculation are related to the harmonic order. Therefore, it is necessary to form the node admittance matrix of the network multiple times according to the harmonic order. Due to the particularity of the harmonics, it is necessary to form according to the phase sequence of the subharmonic. The corresponding sequence network.

\section{Research cases}

\subsection{Analysis of harmonic resonance mechanism of PV power plant based on harmonic power flow method}

Researching on harmonic overvoltage mechanism of multi-power electronic grid-connected system by harmonic current flow method, the equivalent impedance of the harmonic network of a PV power plant in Qinghai Province is shown in Fig. 3.

\subsection{Scenario 1: The influence of the impedance of PV inverter on the resonant frequency}

Two sets of PV inverters' impedance parameters are set to analysis the influence of inverter parameters on the harmonic voltage distribution of the node. The harmonic power flow calculation results of PV power plant are shown in Fig. 4.

$>\quad$ Paramters of PV inverter 1: $\boldsymbol{Y}_{\text {con }}=18 \mathrm{~S}$;

$>$ Paramters of PV inverter 2: $\boldsymbol{Y}_{\text {con }}=50 \mathrm{~S}$;

As shown in Fig. 4, when more inverter 2 parameters are used in the system, the frequency and voltage amplification of the high frequency resonance point are significantly increased. When the power generation units in the system all with the same type, there is the only one resonance peak in the range of $0-1000 \mathrm{~Hz}$, and the voltage amplification factor is relatively large.

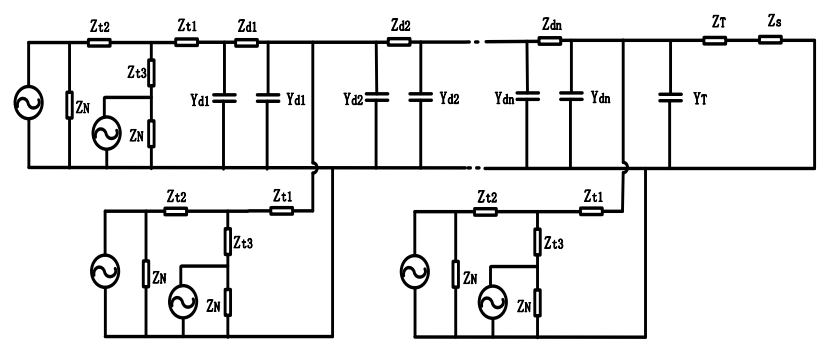

Fig. 3. Network equivalent impedance diagram.

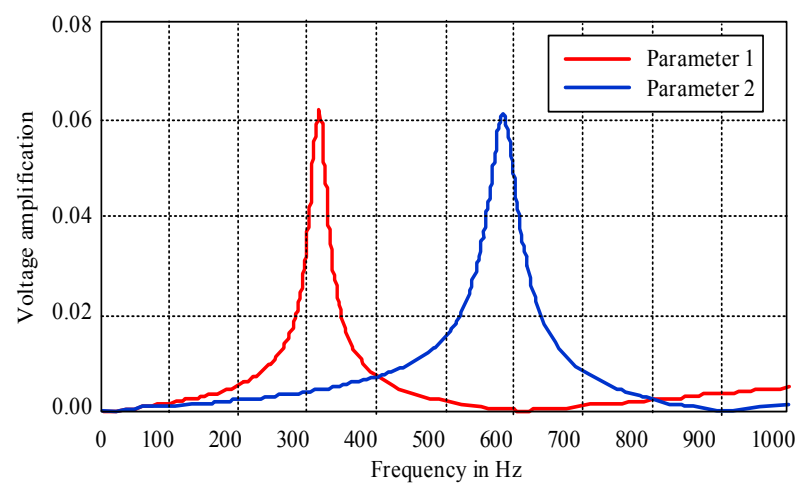

Fig. 4. Influence of converter parameters on harmonic voltage distribution.

\subsection{Scenario 2: The influence of the number of $\mathrm{PV}$ inverters on the resonant frequency}

While the number of inverters running PV power plant is 10,30 , and 50 units, the variation of the harmonic voltage amplification coefficient of the selected node is observed, and the analysis results are shown in Fig. 5.

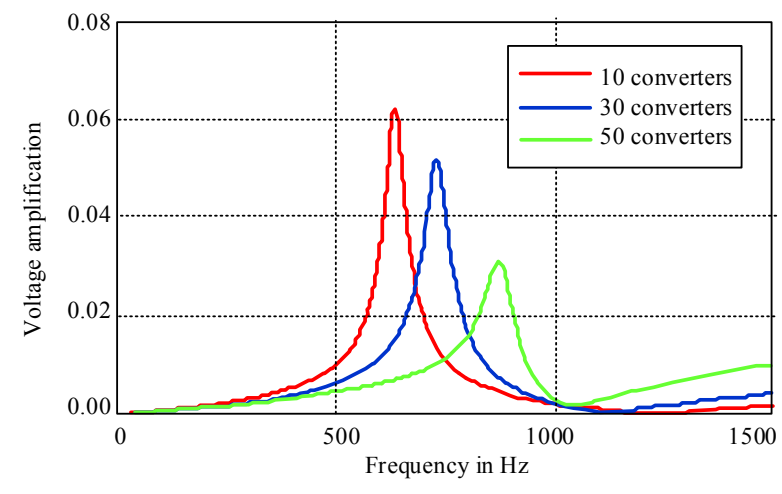

Fig. 5. Influence of the quantity of converters on harmonic voltage distribution. 
As shown in Fig. 5, it is obvious that influence of the input quantity of inverter on the harmonic characteristics of photovoltaic power station. As the number of inverters invested by the PV power plant increases, the resonant frequency of the resonant point with higher frequency gradually increases, the resonant frequency of the lower frequency resonant point gradually decreases.

\subsection{Scenario 3: The influence of grid strengh on the resonant frequency}

The short circuit ratio (SCR) of the system is defined as the ratio between the power system short circuit capacity and the rated capacity of the plant:

$$
K_{\mathrm{SCR}}=\frac{S_{\mathrm{SCR}}}{S_{\mathrm{N}}}
$$

The SCR of PV power plant connected to the grid are set as 3 and 10 respectively, representing the weak grid and the strong grid. The simulation results are shown in Fig. 6.

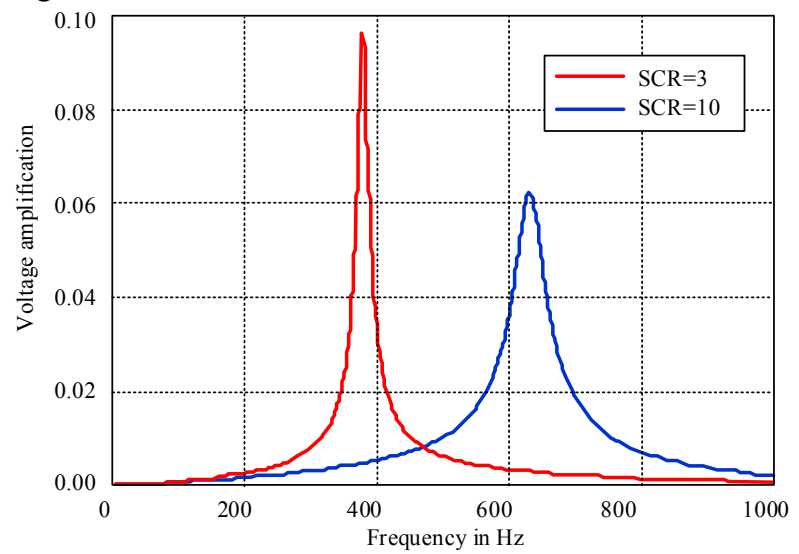

Fig. 6. Influence of power network intensity on harmonic voltage distribution.

As shown in Fig. 6, as SCR increasing, the resonant frequency and the voltage amplification of the higher frequency resonant point increase significantly. For a resonant point with a lower resonant frequency, the resonant frequency increases and voltage amplification factor decreases.
Based on the impedance characteristics of PV inverter, the Norton equivalent model of the PV inverter was derived, which is suitable for harmonic analysis. The harmonic resonance impedance model of PV power plant was established by means of the traditional power system admittance analysis method. Using the harmonic current method, the mechanism of harmonic overvoltage in PV power plants was analysed, and various factors affecting the harmonic resonance point were analyzed, including the converter impedance, the number of converters and the grid strength. The result is used to guide PV power plant to avoid harmonic resonance and cause system failure.

\section{References}

1. X. YUAN, S. CHENG, J. HU. Multi-time Scale Voltage and Power Angle Dynamics in Power Electronics Dominated Large Power Systems [J] . Proceedings of CSEE, 2016,36(19): 5145$5154+5395$ (in Chinese).

2. P. A. Dahono, A control method to damp oscillation in the input LC filter of AC-DC PWM converters [C]. Proc. PESC'02, pp.1630 - 1635, 2002.

3. E. Twining and D. Holmes. Grid current regulation of a three-phase voltage source inverter with an LCL input filter[J]. IEEE Transactions on Power Electronics, vol. 18, no. 3, pp. 888-895, May 2003.

4. W. TONG, S. CHENG, H. XU. Investigation of solution to the non-linear equation setup of SHEPWM in converters $[\mathrm{J}]$. Proceedings of the CSEE, 1998, 18(5) : 357-360(in Chinese).

5. J. GAO, P. LI, J. SUN. Analysis of Resonance Distribution Range and Influence Factors of Clustered Distributed Photovoltaic [J]. Power System and Clean Energy, 2016,32(07): 125-131+148.

6. L. WANG, Z. WANG, B. LU, C. ZHANG. Design of the Controller of the Single Phase PV GridConnected Inverter with LCL Filter [J]. Power System and Clean Energy, 2014,30(12):114-118.

7. X. WANG, G. YAO, L. ZHOU, F. WANG, L. GU, J. WANG. Analysis of Causes of Oscillation in the Wind Farm System Based on DFIG [J]. Power System and Clean Energy, 2017, 33(07): 97-104+109.

\section{Conclusion}

